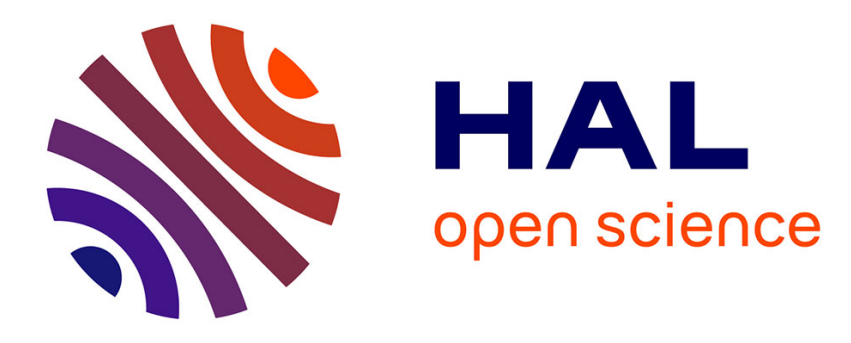

\title{
La double expérience des personnes atteintes d'une maladie neuromusculaire: rétraction et extension
}

\author{
Myriam Winance
}

\section{To cite this version:}

Myriam Winance. La double expérience des personnes atteintes d'une maladie neuromusculaire: rétraction et extension. Sciences Sociales et Santé, 2003, 21 (2), pp.5-30. halshs-00082505

\section{HAL Id: halshs-00082505 \\ https://shs.hal.science/halshs-00082505}

Submitted on 9 Aug 2006

HAL is a multi-disciplinary open access archive for the deposit and dissemination of scientific research documents, whether they are published or not. The documents may come from teaching and research institutions in France or abroad, or from public or private research centers.
L'archive ouverte pluridisciplinaire HAL, est destinée au dépôt et à la diffusion de documents scientifiques de niveau recherche, publiés ou non, émanant des établissements d'enseignement et de recherche français ou étrangers, des laboratoires publics ou privés. 


\title{
La double expérience des personnes atteintes d'une maladie
}

\author{
neuromusculaire : rétraction et extension.
}

\author{
Myriam Winance
}

Paru dans Sciences Sociales et Santé (juin 2003), vol. 21, n², pp.5-30.

E-mail : winance@vjf.cnrs.fr

Dernière version du texte avant publication.

[Note : la numérotation des pages correspond à celle de l'article publié. Mais il peut exister certaines différences mineures entre ce texte et le texte publié]

Résumé.

Partant de l'expérience des personnes atteintes d'une maladie neuromusculaire, l'auteur ${ }^{1}$ montre que la maladie et le recours à des dispositifs d'aide ne s'analysent pas seulement comme une modification du soi (une rupture biographique) mais comme une transformation des liens définissant la personne, son corps et son monde. La maladie provoque un mouvement de rétraction: le repli de la personne est retrait du monde. L'ajustement, matériel et émotionnel, avec le fauteuil roulant provoque un mouvement d'extension. Cette analyse conduit à la suspension des frontières corporelles et personnelles. L'ajustement est un mouvement à la fois d'ouverture et de fermeture de ce qu'est la personne.

Mots-clés : maladie chronique, aide technique, soi, personne

\footnotetext{
${ }^{1}$ Je remercie V. Rabeharisoa pour ses commentaires et encouragements lors de cet article et tout au long de ma recherche. Je remercie également la personne ayant réalisé le référé de cet article, dont les commentaires m'ont permis de creuser mon argument pour ce texte, mais aussi pour la suite de mes recherches.
} 


\section{La double expérience des personnes atteintes d'une maladie neuromusculaire : rétraction et extension.}

Depuis les années 1970, la sociologie de la santé s'est penchée sur l'expérience des personnes devant vivre avec les conséquences soit d'une maladie chronique, soit d'un traumatisme passé. Différents aspects de cette expérience ont été analysés : l'impact de la maladie sur la vie quotidienne de la personne et de son entourage, les stratégies mises en place par ces acteurs pour faire face à la maladie (Baszanger, 1986; Strauss, 1992 ; Waissman, 1995), et l'analyse de l'expérience vécue de la maladie ou du handicap, dans sa dimension identitaire (Charmaz, 1983, 1999; Kelly, 1992; Ville et Ravaud, 1994). Pour analyser la dimension identitaire de l'expérience vécue de la maladie, les auteurs utilisent les notions de « soi » (ou d'identité subjective), «d'identité sociale» et «d'image du corps ». La notion de « soi » se réfère à l'image (idée ou représentation) que chaque individu forme de lui-même à partir de ses expériences, de ses sensations, de ses actions et de ses interactions quotidiennes ${ }^{2}$. La notion «d'identité sociale » se réfère à la représentation ou à l'idée que les autres forment du sujet, lors de leurs interactions, à partir des attributs possédés par ce sujet. Enfin, la notion « d'image du corps » se réfère à l'image que l'individu forme de son corps tout au long de son histoire, à partir de la manière dont il perçoit et ressent son corps, mais aussi à partir des représentations et des significations sociales et culturelles du corps. A travers son histoire et sa vie quotidienne, chaque individu acquiert une représentation de ce qu'il est, comprenant une image de son corps et correspondant ou non à la manière dont les autres le définissent ; cette représentation est basée sur des perceptions et expériences concrètes. Or, la maladie chronique modifie ces expériences concrètes, elle modifie le corps de la personne, ce que cette personne est capable de faire, ses relations aux autres, etc. Elle provoque dès lors l'apparition d'un double décalage. Premièrement, elle provoque un décalage au niveau personnel : ce que la personne est devenue ne correspond plus à l'image qu'elle avait formée d'elle-même. Deuxièmement, elle provoque un décalage au niveau social : ce que la personne est devenue ne correspond plus à l'idée que les autres avaient formée d'elle. D'où l'idée selon laquelle l'expérience de la maladie chronique constitue une rupture biographique (Bury, 1982) ou une perte de soi qui engendre une souffrance particulière (Charmaz, 1983, 1999; Murphy, 1990; Yoshida, 1993). Par différentes stratégies, la personne cherche alors à résoudre ces décalages créés par la maladie. Elle peut soit maintenir la rupture (maintenir son identité passée

\footnotetext{
${ }^{2}$ Cette définition, que je reprends à Kelly (1992), résume les conceptions du soi de différents auteurs. Dans son article, Kelly réalise une liste de ces définitions. Il réalise le même travail pour la notion d'identité dont il n'existe pas non plus une définition unique.
} 
malgré les transformations provoquées par la maladie), soit rétablir une unité en transformant l'image de son soi et de son corps.

Dans l'analyse de cette expérience, certains auteurs (Seymour, 1998) ont mis en évidence le lien entre le corps et le soi. Ils ont montré que le corps constitue le fondement existentiel du soi (Williams et Bendelow, 1998), en utilisant la notion, tirée de la pensée de Merleau-Ponty (1971), «d'embodiment» ou, en français, de «conscience incarnée », selon laquelle le corps est sujet de la perception et par conséquent, est notre accès au monde ${ }^{3}$. L'homme ne perçoit et n'agit qu'à travers et grâce à son corps. Le corps lui permet de percevoir et d'agir, mais cela, parce que, dans la vie quotidienne, il n'est pas lui-même perçu : il se retire. Le corps est vécu sous le mode de l'être : le corps est sujet. La maladie chronique, au contraire, rend le corps éminemment présent; celui-ci devient le centre des préoccupations ; il est vécu sous le mode de l'avoir. Un décalage s'opère entre le sujet et son corps. L'image que la personne a de son corps ne correspond plus à ce que son corps est, celui-ci est devenu une présence étrangère (Leder, 1990). La notion de «dys-embodiment» signifie que le corps est présent comme dysfonctionnant. La personne est alors engagée dans un processus de reembodiment (Williams et Bendelow, 1998) ou de réhabilitation (Seymour, 1998). Celui-ci consiste essentiellement dans un travail sur l'image du corps, travail qui aboutit à la réunification du corps et du soi. Seymour analyse comment à travers les pratiques corporelles quotidiennes, la personne est amenée à transformer l'image qu'elle a de son corps et arrive à reconstruire son soi incarné.

Ces travaux abordent l'expérience de la maladie en prenant comme points d'entrée les notions de soi, d'identité sociale et d'image du corps, trois notions définies en termes d'image ou de représentation. Leur analyse privilégie dès lors la dimension cognitive et intime (intérieure) de l'expérience de la maladie. La maladie est décrite comme produisant un décalage entre la réalité -le corps- et la représentation -l'image du corps, l'image de soi par soi, l'image de soi par autrui. La reconstruction de la conscience incarnée est réalisée à travers un travail identitaire, analysé comme un processus cognitif interne de transformation de l'image de soi et de l'image du corps, transformation à travers laquelle le soi et le corps sont réconciliés. De même, lorsque ces travaux se focalisent sur l'expérience vécue et les transformations du corps, ils analysent la confrontation entre deux corps : «le corps réel » et « le corps représenté » ou «imaginé », cette confrontation étant une épreuve pour la personne (Morin et Salazar-Orvis, 1996), vécue à l'intérieur d'elle-même, dans son

\footnotetext{
${ }^{3}$ Pour Merleau-Ponty, le corps est ouverture au monde. Il est aussi ce qui lui donne sens à travers la perception (Merleau-Ponty, 1971).
} 
« soi ». Que l'accent soit ainsi mis sur la dimension cognitive et « intérieure » de l'expérience de la maladie et du processus d'embodiment est lié à la méthode utilisée : le récit de vie ou l'entretien. A travers ceux-ci, la personne donne sens et cohérence à son expérience; elle se raconte. Son discours rend « son soi » perceptible.

Dans cet article, mon objectif est de reprendre cette question de l'expérience de la maladie et celle de l'embodiment, en prenant comme point d'entrée non le soi, mais le corps. L'expérience de la maladie engage la personne non seulement dans un processus cognitif et intérieur de transformation, mais aussi dans un processus de transformation corporelle. Les personnes ayant eu un accident ou atteinte d'une maladie neuromusculaire décrivent leur expérience comme étant d'une part celle d'un corps constamment transformé par la maladie (ou transformé par l'accident) et d'autre part, celle d'un corps qui ne peut (sur)vivre et agir que grâce à des techniques qui, elles aussi, transforment le corps. Dans cette expérience, la transformation du corps, par la maladie ou par des techniques, est importante. Mon objectif est de décrire cette transformation corporelle en tant que telle, et d'analyser le processus de reconstruction du soi incarné à partir de cette double expérience de transformation du corps.

Pouvoir analyser et décrire l'expérience de la maladie et le processus d'embodiment en mettant l'accent sur la transformation corporelle, deux déplacements sont nécessaires; le premier est méthodologique, le second est théorique. La méthode utilisée pour le recueil des données a été l'observation. Si le soi est perçu à travers le récit, le corps, lui, est perçu à travers l'observation. Le soi se raconte et s'écoute, le corps se met en scène et s'observe. Les observations sur lesquelles cet article est basé sont tirées de deux terrains différents. Le premier terrain a consisté dans le suivi, durant deux mois, de deux Services Régionaux d'Aide et d'Information (SRAI) de l'Association Française contre les Myopathies. Ces services (composés d'un directeur, une secrétaire et trois ou quatre techniciens d'insertion) ne sont pas des services de soin ; ils aident et informent les familles dont l'un des membres est atteint d'une maladie neuromusculaire, pour tout problème lié à cette maladie ${ }^{4}$. Lors de cette enquête ethnographique, j'ai observé vingt-cinq visites à domicile. Le deuxième terrain a consisté dans l'observation d'essais de fauteuils roulants se déroulant dans un centre d'essais spécialisé. J'ai observé trente-quatre essais de fauteuils roulants, sur une période de 9 jours. Les personnes observées étaient

\footnotetext{
${ }^{4}$ Questions touchant la scolarité, le travail, l'information sur les maladies, les aides techniques ou humaines, l'accessibilité, le transport, etc... Ils ont également un rôle d'information sur les maladies neuromusculaires auprès des professionnels médico-sociaux. (voir Rabeharisoa et Callon, 2000).
} 
soit atteintes d'une maladie neuromusculaire, soit atteinte d'une pathologie autre, soit, encore, avaient subi un accident.

Le deuxième déplacement réalisé est d'ordre théorique. L'analyse de la transformation corporelle suppose de suspendre, pour un temps, l'intégrité corporelle de la personne ; elle suppose de mettre en question le corps comme entité matérielle définie, ce que ne fait pas la sociologie de la santé. Celle-ci prend acte de la transformation du corps, analyse ses conséquences, mais n'analyse pas le processus en lui-même ${ }^{5}$. Je mobiliserai la sociologie des techniques appelée théorie de l'acteur-réseau (Akrich, 1987 ; Callon, 1986 ; Clarke, 2000 ; Latour, 1993). Le constat de départ cette sociologie est que les sciences sociales ont malmené les objets soit en les ignorant, soit en les expliquant par leur fonction sociale (Latour, 2000b). Son objectif est, dès lors, de prendre en compte et d'expliquer l'objet (technique ou scientifique) en tant que tel. Son premier apport, utile pour la sociologie de la médecine, est la prise en compte du non-humain et de sa matérialité à côté de celle de l'humain et du sens. Son deuxième apport réside dans la manière dont elle a abordé l'objet. En analysant le processus d'innovation, elle a déployé l'objet et a montré que celui-ci n'existait que parce qu'il était lié à d'autres entités humaines ou non-humaines. La notion de réseau désigne l'ensemble de ces relations qui font exister et qui font tenir l'objet. La matérialité, la résistance, l'objectivité et la spécificité de chaque objet sont façonnées par ces relations. De cette notion de réseau, découle une théorie de l'action comme distribuée entre des entités associées les unes aux autres et comme une propriété émergente du réseau. Le dernier apport de cette sociologie est sa méthode d'enquête qui suppose une description et un suivi minutieux de l'objet afin, justement, d'observer et de saisir les relations qui le font.

La manière dont la sociologie des techniques a pris en compte l'objet en tant que tel peut être utilisée, en sociologie de la médecine, pour prendre en compte le corps en tant que tel dans sa matérialité, pour déployer ce qu'est ce corps, et pour décrire la manière dont il est transformé par la maladie ou les aides techniques. De la même façon que la sociologie des techniques a décrit l'objet comme façonné à travers un réseau hétérogène de relations, je décris le corps comme fabriqué à travers un ensemble de liens. Cette approche me permettra d'analyser la transformation corporelle vécue par la personne. Le déploiement de cette transformation me permettra, d'une part, de comprendre l'émergence de l'action

\footnotetext{
${ }^{5}$ Strauss (1992), par exemple, analyse l'impact de la technologie sur la gestion de la maladie et sur sa trajectoire. Waissman (1995) analyse son impact sur les interactions entre le malade et ceux qui l'entourent. Mais aucun des deux ne met en question ce qu'est le corps.
} 
(«comment une personne acquiert-elle des capacités et des incapacités ? ») ${ }^{6}$, et, d'autre part, aboutira à une définition particulière de la personne, non en termes de soi, mais en termes de relations. Cela permet de mettre en évidence non pas l'aspect intime de l'expérience de la maladie, mais son aspect «mondain $»^{7}$. Dans la suite du texte, je montrerai comment la personne atteinte d'une maladie neuromusculaire ou ayant subi un accident, est définie par un double mouvement de rétraction et d'extension.

\section{La maladie comme rétraction : repli et retrait}

Les maladies neuromusculaires sont des maladies dégénératives, pour la plupart d'origine génétique, qui se traduisent par la perte progressive des capacités fonctionnelles et, selon les cas, par une atteinte des fonctions vitales. Elles se déclarent à différents âges de la vie et sont plus ou moins invalidantes. Actuellement, il n'existe aucun traitement curatif, mais uniquement des possibilités de prise en charge pour lutter contre les effets de la maladie. Voici un extrait tiré d'une visite à domicile ${ }^{8}$ avec le SRAI chez un couple dont l'homme est atteint d'une dystrophie myotonique de Steinert ${ }^{9}$.

Directeur (en regardant Monsieur Georges) : Vous pouvez raconter un peu votre parcours ?

Monsieur Georges : Et bien... la maladie s'est vraiment développée à partir de 30 ans. Avant cela allait.

Madame Georges : oui, il a commencé à avoir des problèmes cardiaques. Moi, je voyais qu'il marchait doucement. Mais bon... [...] Au début de la maladie, il pouvait encore marcher, difficilement mais il marchait. Mais au point de vue cardiaque, cela s'est vite dégradé, il a fait un infarctus et depuis, cela ne va plus. Il ne peut pas faire un effort.

Monsieur Georges : et maintenant, j'ai une pile.

\footnotetext{
${ }^{6}$ Les travaux de sociologie de la santé centrés sur le soi permettaient d'expliquer le sens de l'expérience pour la personne.

${ }^{7}$ Au sens de Merleau-Ponty pour qui le corps est toujours relation au monde.

${ }^{8}$ Il s'agit ici d'une première visite, réalisée par le directeur du SRAI et un technicien d'insertion; les visites suivantes seront réalisées par le technicien d'insertion seul.

${ }^{9}$ La dystrophie myotonique de Steinert est une maladie génétique autosomique dominante associant une dystrophie musculaire (atrophie des muscles), une myotonie (retard au relâchement musculaire), à des anomalies d'autres organes (œil, système nerveux, appareil cardio-respiratoire...). Elle touche essentiellement l'adulte.
} 
Directeur : vous portez un pacemaker?

Monsieur Georges : oui, et puis il y a les yeux aussi, j'ai été opéré de la cataracte. Mais cela a fort baissé depuis. J'ai du mal pour voir. Cela a empiré.

Madame Georges : oui... enfin... c'est tout... il y a tout qui ne va pas. Tout s'est dégradé... quand il a besoin de quelque chose, il me demande, parce qu'il ne peut pas le faire... je le fais pour lui. Et quand je ne suis pas là... et bien, il ne peut rien faire. Il a toujours besoin de moi. [...] C'est pour tout pareil... La journée, il ne sait rien faire. Dans la vie quotidienne, tout devient impossible.

Monsieur Georges : je suis allongé ici toute la journée... quand ma femme est partie.. je regarde la TV et je ne peux rien faire.

Madame Georges : il ne peut rien faire, quand je m'en vais, (elle montre la bouteille d'eau sur la table) je lui ouvre la bouteille, je lui mets la paille et le verre sur la table et il se débrouille pour boire s'il a soif. Parce qu'il ne peut pas porter le verre à sa bouche... il ne peut rien prendre... ses mains restent raides (Monsieur Georges montre ses mains et explique qu'il ne peut plus plier les doigts).

Directeur : le fait que vos mains soient raides, c'est parce que vous ne pouvez plus contracter vos muscles.

Madame Georges : et même ses muscles fondent. Il n'a plus de muscle. Là, cela ne se voit pas, parce qu'il est habillé, mais quand je le lave... [...] Mon mari, il voudrait bien sortir, mais bon, comme cela, ce n'est pas possible. Mon mari, c'était quelqu'un de bien remuant, il jouait à la pétanque, aux cartes, c'est sûr que maintenant, il est bloqué. Parce que, c'est bien les copains, mais personne ne vient le voir. Et lui, il ne peut plus sortir, il ne peut plus se déplacer.

(SRAI, Monsieur et Madame Georges, décembre 1998)

Dans cet extrait de la conversation, Monsieur et Madame Georges décrivent l'action de la maladie. La maladie agit sur le corps de Monsieur Georges, elle déforme son corps qui perd peu à peu ses capacités. Le corps ne peut plus se tenir droit, il devient raide et faible. Il devient progressivement immobile. La maladie prive le corps de sa mobilité et de sa souplesse. Les rétractions et les déformations musculaires sont de plus en plus nombreuses, de sorte que la personne devient immobile non seulement parce qu'elle ne peut plus bouger, mais aussi parce qu'elle ne peut plus être bougée. La maladie transforme le corps en une masse inerte, lourde et rigide. Elle lui donne une forme. Le corps devient une matérialité douloureuse et rigide qui ne peut plus bouger. Cet extrait montre que cette transformation du corps est simultanément une transformation du monde de Monsieur. La réduction de son corps est retrait du monde. Plus la 
maladie évolue, plus Monsieur Georges perd de capacités et plus son monde se rétrécit. La perte de la mobilité est une réduction du monde: l'ensemble des liens qui existaient entre Monsieur Georges et d'autres humains ou non-humains se défait. Ces liens se raréfient. Avant, Monsieur Georges travaillait, il allait jouer aux cartes avec des amis, maintenant, «Tout devient impossible ». Le monde de Monsieur se réduit à son corps, à la survie de son corps. Pour qualifier ce processus de transformation simultanée du corps et du monde de la personne, j'emploie un mot issu du vocabulaire médical, celui de « rétraction ${ }^{10}$. Rétraction comprend l'idée de «retrait» et celle de «repli » ou « recroquevillement». Peu à peu, la personne se rétracte sur son corps, elle se recroqueville. Mais cette rétraction de la personne est simultanément «recroquevillement » du corps et retrait du monde. La transformation du corps en une matérialité rigide est aussi pour la personne une réduction de son monde.

Scarry (1985), dans ses travaux sur la torture, a donné une analyse de ce mouvement conjoint de réduction du monde et du corps à travers la douleur. Pour analyser ce mouvement, elle opère un rapprochement entre douleur et langage. Elle part d'un double constat : d'une part, celui de la difficulté que le sujet ressent à exprimer sa douleur et d'autre part, celui d'une opposition entre la certitude de la douleur pour celui qui la ressent et l'incertitude sur la douleur de l'autre (celui qui écoute ne peut jamais être certain de la douleur de l'autre). La douleur, nous dit-elle, résiste à toute tentative d'objectivation et de saisie par le langage car la douleur est un état intentionnel pur, sans objet, ou plus précisément, un état de passivité pure (car un état intentionnel n'est intentionnel que par l'objet visé). La douleur déconstruit le monde du sujet et l'enferme dans son corps en coupant toute possibilité de lien entre un sujet et un objet, en interdisant toute expansion du sujet dans le monde ${ }^{11}$. La douleur est destructrice car elle a un pouvoir de « dé-objectivation », elle détruit tout objet et produit un pur sujet, un pur sujet parce que réduit à l'état de «sentant». Ce bref rappel de l'analyse de Scarry permet d'expliquer pourquoi le repli du corps est retrait du monde. La maladie, à travers la douleur, touche la capacité de la personne à faire lien et à être liée, elle touche la mobilité de la personne: sa capacité à se mouvoir et à être mue. Pour Scarry, le corps douloureux est réduit à lui-même. Ce corps douloureux, fermé et rétracté,

\footnotetext{
${ }^{10}$ Ce mot désigne, dans le champ médical, un raccourcissement pathologique d'un organe ou d'un tissu (Larousse Médical).

${ }^{11}$ Leder (1990) a montré également que la douleur oblige le sujet à focaliser son attention sur son corps douloureux et produit ainsi une rupture entre le sujet, le monde et autrui.
} 
est devenu une matérialité rigide ; matérialité rigide parce qu'elle est incapable de s'étendre et de créer des liens avec des entités extérieures. Or, ce sont ces liens qui lui permettent d'exister (Latour, 2000a). Dans les liens qu'il tisse avec le monde, le sujet acquiert sa capacité d'action. Scarry met en évidence que le sujet ne peut vivre que s'il est lié.

Monsieur Georges est peu à peu réduit à une matérialité rigide car l'ensemble des liens qui l'unissaient au monde extérieur se raréfient. La dégradation du corps est simultanée au dépeuplement du monde. Dans la conversation avec le SRAI, un élément revenait à plusieurs reprises : la question du mouvement et de la mobilité. La maladie agit sur la mobilité, elle rend le mouvement impossible ou difficile. Non seulement Monsieur et Madame Georges ne se déplacent plus ou peu à l'extérieur, mais l'extérieur ne se déplace plus chez eux. Monsieur Georges était quelqu'un de bien remuant : il jouait aux cartes et à la pétanque, et avait de nombreux amis. Maintenant, Monsieur Georges est couché toute la journée sur son divan, il regarde la télévision et est seul. "C'est bien les copains, mais personne ne vient le voir. Et lui, il ne peut plus sortir, il ne peut plus se déplacer.» (Madame Georges). La maladie rend immobile, elle rend tout déplacement, tout lien, impossible. Elle agit sur la mobilité : sur le «faire lien ». Cette destruction du mouvement par la maladie est double, puisque d'une part, la personne ne peut plus se mouvoir dans le monde et d'autre part, le monde ne meut plus (voire, ne mobilise plus) la personne. Pour le dire autrement, la personne n'émeut plus le monde, et le monde n'émeut plus la personne. Comme l'a montré Despret (1999), le corps ému est un corps mis en mouvement par le monde. La « rétraction » de la personne et de son monde est opérée à travers la perte du mouvement ou mieux la perte de la mobilisation. Le corps devient douloureux car il devient immobile, incapable de se mouvoir ou d'être mu.

La perte du mouvement, mouvement fonctionnel mais surtout émotionnel, est réduction simultanée du corps et du monde. La personne perd la capacité de s'étendre. A un moment, Madame Georges dit que son mari est triste. La maladie touche ce qui fait que la personne était et est elle-même. La modification du monde et du corps est une modification de la personne. La maladie, en agissant sur l'ensemble des liens qui définissent la personne et qui articulent son corps et son monde, crée la douleur : le repli de la personne sur elle-même et son retrait du monde. La maladie apparaît, dans mon analyse, comme touchant la mobilité de la personne et la manière dont elle est mobilisée par d'autres entités, comme touchant sa capacité à faire lien et à être liée, capacité matérialisée par le corps. Il est alors étonnant d'observer la mobilité et la vivacité des enfants atteints d'une maladie, lors des rassemblements de l'AFM. Comment toutes ces personnes sont-elles devenues mobiles, alors que la maladie les rend immobiles ? 


\section{L'interaction avec le dispositif technique comme extension}

Répondre à cette question consiste à analyser le deuxième aspect de l'expérience des personnes : la vie avec et grâce à des aides techniques. Pour cela, je décris l'exemple du fauteuil roulant et un moment particulier du choix d'un fauteuil : l'essai d'un fauteuil roulant. David, âgé de 20 ans, est paraplégique suite à un accident de la route. Accompagné de son ergothérapeute et d'une aide soignante, il est au centre d'essai pour choisir un fauteuil roulant manuel. Au début de l'essai, le responsable du centre commence, avec l'ergothérapeute et David - mais celui-ci intervient peu -, par lister les critères du cahier des charges. Le choix du fauteuil doit être guidé par un certain nombre de contraintes: le prix (pas trop élevé), des repose-pied amovibles (l'ascenseur permettant à David d'accéder à son appartement est court), pliant pour rentrer dans la voiture, performant pour faire du deux-roues. Sur base de ces critères, David a repéré un fauteuil traditionnel. Mais le responsable l'invite à essayer un autre fauteuil, un fauteuil léger.

Responsable : l'important, pour un jeune paraplégique actif, c'est d'avoir un fauteuil roulant manuel maniable, léger et costaud (il a sorti un fauteuil léger haut de gamme). Quand on a des repose-pied escamotables, les jeunes actifs, ils font peiner le fauteuil, ils vont partout avec... et le fauteuil, il ne tient pas, il casse. Lorsque l'on a des repose-pied fixes, le fauteuil est beaucoup plus rigide et plus costaud. [...] (Le responsable retourne le fauteuil pour régler la profondeur et le dossier à la taille de David.)

David : et le dossier... il sera trop bas ?

Ergothérapeute : tout est réglable. Pour l'ascenseur, cela ira?

(David se place parallèlement au fauteuil pour comparer les longueurs et explique que dans son fauteuil actuel, lorsqu'il a retiré les repose-pied, il reste tout juste 2 ou $3 \mathrm{~cm}$ dans l'ascenseur.)

Responsable (il change les roues du fauteuil; au lieu de roues avec des pneus épais, il place des roues dont les pneus sont très fins) : voilà, pour le look, cela devrait être mieux. (Centre d'essais, David, mai 1999).

A travers la confrontation entre David et le fauteuil, le responsable écarte pour un temps les contraintes définies au départ dans le cahier des charges. Dans l'extrait ci-dessus, il écarte la contrainte «repose-pied amovibles» pour faire émerger la question de l'activité de David et de la solidité : si David veut être actif, il doit choisir un fauteuil dont la palette-repose-pied est fixe, sinon, son fauteuil risque de casser rapidement. Il fait également apparaître le critère de l'esthétique. Par ce travail, 
le responsable cherche à ouvrir des possibilités pour David. Si David choisit l'un ou l'autre fauteuil, ses capacités et son monde ne seront pas les mêmes. Dans un cas, il sera actif, dans l'autre, il devra faire attention. Durant la séance, le responsable fera essayer quatre fauteuils à David: trois sont des fauteuils légers et un est un fauteuil de sport. A travers cette confrontation entre David et les différents fauteuils, le responsable tente de modifier les contraintes posées au départ pour ouvrir le monde de David ou pour ouvrir David au monde. L'enjeu est de rendre David disponible pour le monde et le monde disponible pour David. L'essai apparaît comme un travail sur le monde. Mais, on retrouve, comme dans le cas de Monsieur Georges, le lien corpsmonde. Le travail sur le monde passe par un travail sur le corps de David. L'essai montre que les acteurs se posent la question : «Quel monde émergera pour David ? », en se posant la question «Quel corps pourra-t-il être et avoir dans ce fauteuil ? ». L'extension de la personne, sa possibilité de retrouver sa mobilité, dépend de la manière dont elle est liée à son fauteuil. L'essai de fauteuil apparaît comme un travail sur les liens. Ce travail sur les liens est d'une part un ajustement matériel et d'autre part un ajustement émotionnel.

\section{Ajustement matériel}

David vient d'être installé dans un fauteuil léger pliant actif. Il s'installe correctement sur le fauteuil, place ses jambes sur la palette-repose-pied et glisse un mousse entre ses deux genoux pour qu'ils ne se cognent pas l'un l'autre. Il fait quelques mouvements dans la pièce.

Ergothérapeute : cela te fait drôle car sur ce fauteuil-là, tu es assis différemment par rapport à ton fauteuil. Sur ton autre fauteuil, tes pieds sont beaucoup plus en avant. [...] Tu te sens mal à cause du dossier?

David : oui.

Ergothérapeute : son dossier à lui est un peu plus haut. Il n'y a pas moyen d'avoir un dossier plus haut?

Responsable (il regarde dans sa documentation) : non, c'est une taille unique. Mais moi, je ne suis pas sûr que cela n'aille pas. (Il passe sa main sur le dossier pour évaluer où arrive le dossier par rapport au dos de David). Il est habitué à avoir un dossier haut, mais si on peut le supporter, c'est mieux d'avoir un dossier un peu plus bas. Bon, il faut qu'il se remuscle un peu le dos.

Ergothérapeute : oui... il n'est pas habitué, son accident est récent, son dos doit se remuscler, il faut du temps. 
Responsable (il passe ses mains entre le fauteuil et les jambes de David) : oui... là, il est un peu serré au niveau de la largeur. D'habitude, j'aime bien lorsque c'est étroit, mais là, c'est trop juste.

Ergothérapeute : ce n'est pas possible d'enlever cela (elle montre les protège-vêtements) ?

Responsable : non, c'est pour les roues. Là, il est trop à l'étroit, cela va frotter et appuyer, à force, cela risque de faire une escarre... Il n'y a pas de problème de rougeur ?

Ergothérapeute : non.

David : non.

Responsable : mais bon, à force, cela pourrait venir. Non, il faut prendre une taille au-dessus, 40, ce sera bien... On pourrait peut-être remonter le dossier ? Mais... le sien est plutôt haut, il faudrait voir à combien il est (il mesure). Il n'y a pas beaucoup d'écart, mais comme la pente est plus importante sur le sien...

David : oui, ici, j'ai l'impression d'être assis plus droit, que cela fait un angle droit.

Ergothérapeute : oui, tu es assis plus droit.

David : ah... c'est pour cela que je me sens moins bien.

(Centre d'essais de fauteuils roulants, David, mai 1999).

Durant l'essai, les acteurs cherchent à faire émerger « la meilleure position » c'est-à-dire une position confortable pour David, qui rendra possibles pour lui certains gestes. Ce processus est lent ; différentes positions sont testées. Les acteurs tâtonnent: ils cherchent en touchant. L'essai est une recherche collective à laquelle prennent part le patient, les personnes qui l'entourent et le fauteuil. Cette recherche collective prend la forme d'une double confrontation et négociation : d'une part entre les perceptions des différents acteurs et d'autre part entre la personne et le fauteuil. Cette confrontation est d'abord une confrontation entre les perceptions des différents acteurs. Autour de David, l'ergothérapeute et le responsable du centre tournent; ils observent sa position et lui renvoient une image de son corps. A plusieurs reprises, le responsable du centre touche David pour évaluer comment David est dans son fauteuil. A un autre moment, il essaie lui-même le fauteuil. A travers ces différents gestes: toucher, regarder, s'asseoir, a lieu un échange des sensations entre David et les autres acteurs, et en circulant entre les acteurs, les sensations sont à chaque passage un peu modifiées. Dans l'échange des perceptions entre David, l'ergothérapeute et le responsable, ce dernier joue un rôle important, car il opère la traduction des perceptions en différents énoncés. Il mesure, il touche, il traduit le sentiment de David en position du fauteuil et inversement il traduit la position $\mathrm{du}$ fauteuil en sensation de David; il 
traduit aussi ses perceptions en risque de blessures corporelles. L'échange des perceptions est traduction de ces perceptions en différents sentiments. Cette confrontation entre les perceptions des différents acteurs est doublée d'une confrontation entre David et le fauteuil. David centre son attention sur la manière dont il se sent dans le fauteuil ; à partir de ses sensations, soit il change de position, par exemple, il déplace ses jambes, soit il demande aux autres acteurs de modifier la position du fauteuil : la hauteur ou l'inclinaison du dossier, etc...

A travers cette double confrontation, les acteurs essaient de définir et d'enrichir la perception que David a de son corps, ils essaient de définir quel corps ce fauteuil façonne, quel corps il lui permet d'avoir et d'être, et si ce corps peut faire surgir de nouvelles possibilités d'actions. Ils cherchent à faire surgir un corps différent, un «corps-dans-un-fauteuil » capable d'agir. Peu à peu, par ce travail, le fauteuil et la personne sont transformés et forment quelque chose de nouveau. Cette transformation est une (trans)formation de leur matérialité. Le fauteuil est peu à peu transformé. Le fauteuil roulant n'est pas un objet fini, mais un objet en devenir ${ }^{12}$ qui évolue avec la personne. Cette transformation continue du fauteuil apparaît dans l'exemple de David. La position des accoudoirs et du dossier est constamment modifiée. Le fauteuil est continuellement remodelé à travers l'essai, puis tout au long de l'usage. On rajoute un cousin, on bricole un système pour soulever les jambes... Progressivement, le fauteuil deviendra le fauteuil de David. Sa matérialité est adaptée à celle de David. Après quelques années de vie commune avec une personne, un fauteuil est souvent méconnaissable tellement il a été bricolé et adapté ${ }^{13}$. Si quelqu'un d'autre que la personne tente de s'asseoir dans ce fauteuil, elle s'y sentira très mal. David est également transformé. Il prend une position différente.

A travers cette négociation collective qui est un travail sur le corps de la personne et sur le dispositif qui lui est adjoint, les acteurs agissent sur la matérialité commune de David et du fauteuil, ils la définissent. «Matérialité » désigne ici la force des liens qui tiennent le corps de la personne. «Matérialité » ne désigne ni le corps physique de l'individu, ni le

\footnotetext{
${ }^{12}$ L'innovateur, lorsqu'il conçoit un fauteuil roulant cherche à un concevoir un objet que l'utilisateur pourra modifier. Les fauteuils les plus chers sont ceux où tout est modifiable.

${ }^{13}$ Ce bricolage de l'objet permettant sa singularisation a été souligné par différent auteurs (Thévenot, 1994). Pour Dodier (1993, 1995), la transformation de l'objet technique est l'un des moyens permettant la personnalisation de l'activité et l'attribution de l'action à la personne. Je traite ce point dans la suite du texte.
} 
fauteuil, mais la force et la résistance de leur conjonction ${ }^{14}$. Plus précisément, elle réfère à la fois au corps et au fauteuil qui sont constitués à travers certains liens. Ce travail à travers lequel les acteurs tentent de faire émerger une matérialité commune entre la personne et son fauteuil est le travail d'ajustement matériel.

\section{Ajustement émotionnel}

Lorsqu'une personne est confrontée à un nouveau dispositif, celui-ci est encombrant et gênant : il est de trop. Pour que l'action soit possible, la personne doit percevoir et sentir son dispositif. L'ajustement émotionnel est la dynamique par laquelle la personne se sent et devient «elle-même-avec-ses-dispositifs », et la dynamique par laquelle, à travers cette perception, la personne devient sujet d'actions surprenantes ${ }^{15}$. L'ajustement émotionnel produit une singularisation de l'aide qui devient source de créativité et ouverture au monde. D'encombrante, l'aide deviendra habilitante. Ce processus d'ajustement émotionnel est proche du processus de familiarisation décrit par Thévenot (1994). Le terme «émotionnel » me permettra cependant d'insister sur la redistribution opérée lors de ce processus, entre ce qui est de l'ordre du corps et ce qui est de l'ordre du monde.

Théo, un petit garçon atteint d'une amyotrophie spinale et âgé de deux ans, essaie son premier fauteuil électrique. Le technicien d'insertion $\mathrm{du}$ SRAI lui explique à quoi servent les différents boutons du fauteuil. Théo joue avec l'interrupteur qui allume et éteint un voyant vert. Le technicien lui montre le klaxon et Théo rigole, ils recommencent le jeu plusieurs fois. Puis, les adultes lui montrent qu'il peut avancer ou reculer en utilisant le joystick, ils lui prennent la main et font le mouvement avec lui. Sa mère, se plaçant devant lui, l'appelle pour qu'il vienne à elle. Peu à

\footnotetext{
${ }^{14}$ Nous verrons dans la suite du texte que la matérialité est toujours ambivalente ; d'une part, elle peut être source de confort et d'action, d'autre part, elle peut être source de douleur et empêcher l'action.

${ }^{15}$ Les essais de fauteuils pourraient être comparés aux «arènes des habiletés techniques » définies par Dodier comme des lieux d'épreuve des capacités de l'individu confronté dans ses actes au fonctionnement des objets techniques et placé au carrefour des jugements d'autrui (Dodier, 1995, p. 220). Les essais de fauteuil constituent des arènes des habiletés techniques dans la mesure où il s'agit d'éprouver les capacités de la personne dans un certain fauteuil. Cependant, l'essai n'a pas pour but de révéler les capacités de la personne, mais bien de les façonner, façonnement qui suppose et entraîne une transformation de l'être de la personne, de son corps et de son monde.
} 
peu, Théo fait bouger le fauteuil, mais dans n'importe quelle direction, sans faire attention aux obstacles. Les adultes doivent prévoir ses mouvements et le forcer à lâcher le joystick à l'approche d'un meuble ou de l'un d'eux pour stopper le fauteuil. A la fin de la séance, Théo commence à comprendre qu'en poussant le joystick, il avance et en le tirant vers l'arrière, il recule. Sa mère lui montre qu'avec son fauteuil, il peut aller partout où il veut. Dans sa chambre, Théo nous dit que ce soir, il dormira avec son fauteuil. Enfin, lorsque le technicien lui demande s'il ira se promener dehors, Théo lui répond qu'il ira au parc courir derrière les canards. A travers l'essai du fauteuil, ont lieu une distribution des compétences et un transfert de l'agir. L'action est en partie prise en charge par la personne et en partie par l'appareil. Ainsi, dans l'exemple de Théo et son fauteuil, «se déplacer » est partagé entre Théo qui bouge le joystick, les adultes qui rectifient son mouvement et le fauteuil qui roule. Lorsque Théo conduira son fauteuil seul, l'action sera distribuée entre lui et le fauteuil. Jusqu'ici, l'interprétation rejoint celle de la sociologie des techniques (Latour, 1993) ${ }^{16}$. Mais l'exemple de Théo fait apparaître la particularité de cette délégation et de cette distribution des compétences. Cette distribution n'est possible que dans la mesure où il y a ajustement matériel. Ensuite, la délégation n'est pas organisée entre les différentes entités. L'action «bouger » est le résultat de la conjonction de multiples petites impulsions venues de partout et qui passent d'un acteur à l'autre. Comme l'ajustement matériel, le processus d'ajustement émotionnel implique les différentes perceptions des acteurs présents, perceptions qui se transforment en actions et inversement : l'un qui guide la main de Théo, sa mère qui l'appelle, le technicien qui explique, le fauteuil qui part, Théo qui appuie sur le joystick. Finalement, Théo «bouge» dans son fauteuil, il ne «se déplace» pas vraiment ou pas encore. Son mouvement est pour l'instant hasardeux et désordonné. Mais à travers les interactions et les échanges de perceptions, l'action est peu à peu attribuée à Théo. Et à travers cette attribution, elle est transformée: « appuyer sur le bouton » devient «Théo joue et rit», «tirer sur le joystick» devient «Théo recule », «bouger» devient «Théo va voir les canards». Le fauteuil n'est plus seulement un fauteuil pour se déplacer, il est médiateur de l'action (Gomart et Hennion, 1999). A travers l'essai, de nouvelles activités et sensations émergent dont le sujet est Théo. Ce processus est l'ajustement émotionnel. A travers les mouvements saccadés du fauteuil, les explications des adultes et ses propres sensations, Théo apprend à sentir le fauteuil, il apprend à sentir le mouvement du fauteuil comme étant son mouvement, comme étant «Théo se dépla-

\footnotetext{
${ }^{16}$ Pour une application de la sociologie de l'acteur réseau aux in/capacités, voir Moser et Law (1999).
} 
çant », et le fauteuil devient son fauteuil avec lequel il ira voir les canards au parc et à côté duquel il dormira ce soir. A travers ce processus, la personne (Théo dans et avec son fauteuil) devient le sujet de l'action, et le lien entre Théo et son fauteuil devient source de créativité: d'actions nouvelles et de nouvelles manières d'aller vers le monde.

Dans ce cas, le processus d'ajustement ne s'opère pas seulement à travers la mise en place de repères appropriés et personnalisés qui rendront possible le maniement habile (Thévenot, 1994), mais à travers « une mise en forme du corps et du fauteuil ». Ce façonnement est matériel et émotionnel. A travers le processus d'ajustement, émerge un corps étendu, c'est à dire à la fois une matérialité, des perceptions et des actions, communes et nouvelles. Dans l'ajustement, la personne apprend à percevoir et à reconnaître les « expressions » du fauteuil ; cette perception rend possible le maniement du fauteuil et l'émergence d'émotions nouvelles, d'où la notion d'ajustement émotionnel. A travers celui-ci, une redistribution est opérée entre ce qui est corps et ce qui est monde ${ }^{17}$. La capacité de la personne à agir et à percevoir est modifiée par le fauteuil. Celui-ci devient ce qui façonne et soutient son corps. L'ajustement entre le corps et le fauteuil est ouverture au monde pour la personne, car il rétablit sa capacité à faire lien. En façonnant les liens entre la personne et le fauteuil, les acteurs façonnent les liens entre son corps et son monde. Ce double travail sur les liens aboutit à une transformation de ce qu'est la personne. Cela apparaît dans l'exemple de David que nous pouvons poursuivre.

David vient d'être installé dans le fauteuil de sport, il rectifie sa position. Puis, il fait une longueur dans la salle, il roule, tourne, fait un peu de deux roues. De temps en temps, il sourit.

Ergothérapeute : cela te gène qu'il n'y ait pas d'accoudoir ?

David : non, mais le fait qu'il ne se plie pas, oui.

Ergothérapeute : il est petit, dans une voiture, cela passe sans problème.

Responsable : oui, la seule difficulté est d'enlever les roues. Mais beaucoup y arrivent sans problème.

Ergothérapeute: tu fais sportif dans ce fauteuil !

\footnotetext{
${ }^{17}$ Despret montre que l'émotion est l'expérience d'une indétermination entre ce qui est corps et monde. Elle est dès lors l'occasion d'une nouvelle distribution, de la fabrication d'une réalité différente. Suchman s'intéresse plus particulièrement au rôle des émotions dans les interactions homme/machine. Pour parler de ces interactions, elle propose le terme de « rencontres affectives » car l'émotion est pour elle un effet de ces rencontres. Elle aussi, met l'accent sur les redistributions opérées dans ces rencontres et sur la modification des frontières entre l'homme et la machine (Schuman, 2002).
} 
Responsable (il passe la main dans le dos de David pour sentir où arrive le dossier) : le dossier est trop bas, je dois le remonter. (Ils font à nouveau un transfert et le responsable remonte le dossier)

Ergothérapeute : cela vaut combien?

Responsable : un peu cher, mais dans son cas, cela vaut la peine de chercher des financements, ce serait dommage de ne pas chercher.

Ergothérapeute : comment fait-on pour plier le fauteuil ?

Responsable (il fait la démonstration): l'astuce, c'est d'enlever les deux roues, puis on replie le dossier sur le siège et c'est tout petit, tout léger, on le glisse soit sur le siège à côté soit entre soi et le volant. Bon, cela fait quand même $7 \mathrm{Kg}$, mais on s'habitue.

Ergothérapeute : oh, tu pourrais le faire !

David: je peux le prendre plus longtemps pour un essai, je peux le prendre pour essayer dans l'ascenseur?

Aide soignante: tu en penses quoi ?

David: cela change !

Ergothérapeute: oui, de tes habitudes, mais quel que soit le fauteuil, cela changera, car ce sera un fauteuil différent du tien!

(Le responsable a fini de monter le dossier, il s'assied dans le fauteuil et roule, il fait du deux roues, sent que les pneus sont plats, les regonfle et règle à nouveau le dossier; puis, il transfère David)

Ergothérapeute : il est beau ! (avec l'aide soignante, elles parlent des couleurs du fauteuil)

David (roule, fait des longueurs, tourne, on le voit sourire): oui...

L'aide soignante: ce qui est bien, c'est que lorsque tu es dedans, on ne voit plus rien, on ne voit que toi. Le fauteuil roulant disparaît... [...]

Responsable: maintenant, la question importante, c'est de regarder l'ascenseur (il prend son mètre et mesure le fauteuil) S'il fait $90 \mathrm{~cm}$, c'est bon, cela passe, sinon... [...]

David: cela fait...

Responsable: quoi ?

Ergothérapeute: cela fait une différence, c'est bien, je trouve. David est souriant, il tourne avec le fauteuil $[\ldots]$.

David: et le dossier, il est comment par rapport au mien ?

Responsable : un peu plus bas, mais on peut régler et puis, pour l'ascenseur, on peut comparer avec le tien (il compare), il n'y a pas beaucoup de différence, il est très court. Pour le dossier, il y a $10 \mathrm{~cm}$ de différence.

David : pourquoi tu rigoles?

Ergothérapeute: tu es convaincu, cela se voit à ton visage...

David : et les freins, on peut avoir des normaux ?[...] Et les couleurs ?

(Centre d'essais, David, mai 1999). 
Dans ce long extrait, nous assistons peu à peu à la transformation de David. A la fin de l'essai, David sourit et pose lui-même les questions, alors que, au début, il avait tendance à laisser son ergothérapeute parler pour lui. Cette attitude passive était traduite dans la conversation entre l'ergothérapeute et le responsable qui employaient le pronom «il » pour parler de lui, au lieu de s'adresser directement à lui. Au début de l'essai, la conversation était indirecte, à la fin, elle devient directe. Cette transformation de David est une transformation à la fois de son corps et de son monde: des liens qui les attachent et les définissent. Ce travail produit une ouverture au monde. Le responsable du service, en proposant à David d'essayer un fauteuil de sport, a écarté pour un temps les contraintes posées au départ, il a fait comme si ces contraintes n'en étaient pas. Dans l'extrait ci-dessus, David et son ergothérapeute reprennent une à une ces contraintes, mais celles-ci sont modifiées: certaines s'effacent (comme le fait que le fauteuil doit être pliant), certaines sont laissées en suspens puis reprises à la fin (comme le problème de l'ascenseur), certaines, enfin, ne sont plus considérées comme des contraintes (c'est le cas du prix). Inversement, d'autres critères sont apparus comme la maniabilité du fauteuil, l'esthétique... A la fin de l'essai, David est souriant et bouge avec son fauteuil. Bien sûr, l'essai ne débouche pas sur une décision définitive. Mais le travail réalisé a permis d'ouvrir de nouvelles possibilités pour David et de rendre possible sa transformation, même si l'on ne connaît pas l'issue de cette transformation.

Dans l'essai, le travail porte sur les liens qui définissent la personne et sur la manière de faire lien. L'ajustement matériel s'accompagne d'un ajustement émotionnel. Le travail sur le corps est un travail sur le monde. Avant l'essai, David était devant une situation fermée, à la fin de l'essai, il est devant une situation qui s'ouvre peu à peu ${ }^{18}$. A la fin de l'essai, David paraît différent; il était silencieux, il est devenu actif; l'aide soignante nous le dit: «Quand tu es dans ce fauteuil, on ne voit plus que toi ! », David-dans-son-fauteuil a laissé la place à David. Son être a été transformé. Cette transformation de son être réfère à la fois à la transformation de son corps (David dans un fauteuil de sport n'est pas le même que David dans un fauteuil traditionnel, son corps est différent dans les deux cas), de ses sensations (il sourit, il est content), de ses capacités (il peut faire du deux-roues et rouler facilement), de la perception que les autres ont de lui (on voit David et non « David-dans-un-fauteuil »). Cette transformation de son être est une transformation de son faire: de sa capa-

\footnotetext{
${ }^{18}$ Dans ce passage, je renforce certains traits. A la fin de l'essai, tout ne devient pas merveilleux pour David. La vie reste difficile pour lui. Mais je veux montrer, en renforçant certains traits, que l'enjeu de l'essai est d'ouvrir des possibilités à la personne et de la diriger vers le monde.
} 
cité à toucher et à être touché par le monde. David retrouve sa mobilité: sa capacité à se mouvoir et à être mû. L'extension de la personne est le résultat d'un double travail d'ajustement: matériel et émotionnel. A travers l'essai, comme travail sur le corps et sur le monde, les acteurs ouvrent les possibilités pour la personne. En modifiant les liens qui la constituent, on modifie son corps et son monde. L'essai de fauteuil est un moment d'ouverture; mais il est, simultanément et corrélativement, un moment de fermeture car les acteurs cherchent à définir les liens qui fabriqueront la personne, et définir, c'est, d'une certaine manière, déterminer. Choisir tel fauteuil, c'est ouvrir certaines possibilités, mais aussi en fermer d'autres. Le responsable fait sentir à David cette ambiguïté : s'il choisit un fauteuil traditionnel, il pourra enlever les repose-pied, mais il ne pourra pas monter des bordures, s'il choisit un fauteuil performant, il pourra «malmener»son fauteuil, mais pas enlever les repose-pied. Cela se traduit émotionnellement pour David que l'on voit sourire, mais dont on perçoit aussi l'hésitation. A travers l'ajustement matériel s'opère un ajustement émotionnel, et celui-ci est lié à une difficulté émotionnelle qui ne disparaît sans doute jamais. L'essai est une épreuve.

\section{Problématiser et fabriquer la personne}

Dans les deux sections ci-dessus, j'ai analysé les deux aspects de l'expérience de la personne de manière similaire, comme une transformation des liens entre son corps et son monde. Dans la première section, j'ai montré que la maladie touchait la mobilité de la personne et la manière dont elle est mobilisée par d'autres entités, touchait sa capacité à faire lien et à être liée. Dans la seconde section, l'interaction avec le fauteuil roulant, à travers la description de l'essai de fauteuil, est également apparue comme un travail sur les liens qui définissent la personne, son corps et son monde.

Cette analyse de la double expérience de la personne conduit à considérer les frontières corporelles et subjectives de la personne. La personne n'est pas d'abord une entité séparée (un corps et un soi), mais un nœud spécifique de liens qui définissent son être, son corps et son monde. Pour désigner cette configuration, j'utilise la notion de singularité. La maladie et l'essai de fauteuil roulant sont des moments au cours desquels ce qu'est la personne est mis à l'épreuve, ou problématisé. La personne est ré-interrogée pour faire surgir ce qui la fait et la constitue. La notion de singularité est l'outil analytique qui me permet de désigner cette ouverture de la personne. Mais le processus d'ajustement est ambivalent. La matérialité commune est à la fois transformée et formée. La personne est à la fois pro- 
blématisée et fabriquée. L'ajustement est constitué d'un double mouvement d'ouverture et de fermeture de la personne. Cette ambivalence du processus apparaît dans l'exemple suivant, celui d'André.

André, âgé d'une trentaine d'années, est atteint d'une dystrophie musculaire de Duchenne de Boulogne et est presque tétraplégique; il lui reste très peu de force dans les mains. Il utilise un fauteuil roulant électrique depuis longtemps, mais avec l'évolution de la maladie, il éprouve de grosses difficultés pour manipuler le joystick, devenu trop dur. André ne se sent plus en sécurité dans son fauteuil et exige que sa mère reste constamment à ses côtés afin de l'aider à conduire le fauteuil et de corriger sa position. Son incapacité à conduire le fauteuil a créé un lien fort entre sa mère et lui ${ }^{19}$. Sa mère doit être constamment présente pour réaliser l'ajustement entre son fils et son fauteuil. Le processus d'ajustement est incorporé par la mère. La singularité est constituée par un ensemble de liens entre le corps d'André, celui de sa mère et le fauteuil roulant. On observe l'émergence d'un corps collectif : les gestes de la mère, son corps, soutiennent et tiennent le corps «d'André-dans-son-fauteuil ». Ce corps collectif rend possible le processus d'ajustement entre André et son fauteuil, et l'émergence d'une matérialité commune qui soit définie et non-finie. D'un côté, la matérialité est définie à travers les liens et par eux, et parce qu'elle est définie, la singularité peut prendre appui sur elle pour agir. D'un autre côté, elle est non-finie : la mère est sans cesse en train d'ajuster André dans son fauteuil, en train de modifier certains liens, pour qu'André reste mobile.

La situation devenant de plus en plus problématique, André et ses parents ont décidé d'essayer un nouveau fauteuil pour pouvoir, grâce à une commande adaptée, redonner à André la possibilité de conduire son fauteuil. Par là, ils mettent à l'épreuve le lien entre André et son fauteuil, ainsi que le lien entre André et sa mère, liens qui le définissent. Cet essai a été pour André une véritable épreuve. Durant deux heures, la mère d'André a essayé d'installer André dans son fauteuil, mais André se sentait toujours mal et en insécurité. Il comparait sans cesse sa position avec la manière dont il est positionné dans son fauteuil.

André a été transféré sur le Twister (le fauteuil).

André : c'est mieux l'assise confort !

Mère : oui, je sais, tu veux l'assise confort.

\footnotetext{
${ }^{19}$ Dans cet article, je n'évoque pas l'aspect collectif de la maladie et des dispositifs d'aide. Sur cette question, voir Callon et Rabeharisoa (à paraître), Hirschauer (1991) et Winance (2000, 2001).
} 
Responsable : l'ennui, c'est que l'on est obligé de prendre le coussin du revendeur et le coussin n'est pas terrible.

Mère : oui, là, tu as le confort de ton coussin. Et ton coussin, c'est moi qui te l'ai fait. Mais bon, un coussin, c'est facile à faire, il suffit d'aller chercher un mousse et de faire une housse.

André : Maman... je vais tomber !

Mère : on te tient! Dès qu'il n'a pas sa table, il panique. C'est parce qu'il n'a rien pour se tenir. (Centre d'essais, André, mai 1999)

L'essai de fauteuil est une épreuve parce qu'il est une recherche collective à travers laquelle on cherche à créer de nouveaux liens pour la singularité. Mais il est aussi une épreuve parce que la singularité doit dénouer les liens qui la faisaient (même si ces liens pouvaient être producteurs d'émotions difficiles ou de douleur). L'essai n'est pas seulement la recherche d'un nouvel «attachement » entre André et un nouveau fauteuil, il est d'abord un «arrachement » entre André et son fauteuil actuel. Le fauteuil qu'il essaie ne ressemble pas au sien. Dès le début de l'essai, sa mère perçoit le malaise et le traduit pour les acteurs présents. Dès le départ aussi, son rôle d'intermédiaire apparaît; c'est à travers elle que la plupart des sensations et remarques d'André parviennent aux autres. Après une heure d'essai, le responsable conseillera à André et à sa famille de ne pas changer de fauteuil, mais d'essayer uniquement d'adapter la commande. A travers l'essai, un seul lien peut être mis en question : celui entre André et le joystick. Le responsable justifiera sa conclusion en disant «André est trop habitué au sien ». A travers l'usage quotidien, le fauteuil a été transformé à un point tel que toute tentative pour dénouer le lien entre André et son fauteuil échoue. Le fauteuil a été modelé pour André. Inversement, André a pris la forme du fauteuil, physiquement et émotionnellement. Le terme d'habitude est ici éclairant car il permet d'insister sur l'aspect corporel du processus ${ }^{20}$. L'ajustement d'André à son fauteuil actuel est tellement fort qu'il est impossible de les détacher. Ils forment une singularité. L'ajustement a fait émerger une matérialité com-

\footnotetext{
${ }^{20}$ Lorsqu'on utilise ce terme, il est difficile d'éviter la référence à Mauss (1950b), à sa notion d'habitus et de techniques du corps. Pour cette raison, les auteurs de la sociologie des usages évitent ce terme qui «rend mal compte de la dynamique de constitution et de révision du réseau de repères formés par l'accointance de la chose à un certain environnement. » (Thévenot, 1994, p.94). Mon objectif est ici de mettre l'accent sur l'idée que le rapport à l'objet modifie le corps de la personne, dans sa matérialité et dans sa relation au monde. Dans le cas du fauteuil, le rapport à l'objet est plus qu'un rapport de familiarité, il est un rapport de constitution.
} 
mune qui est rigide dans la mesure où elle empêche certaines actions et provoque un sentiment de peur, mais aussi une matérialité commune qui fait qu'André est «bien » dans son fauteuil. Le seul ajustement encore possible pour rendre un peu de souplesse à cette matérialité, est celui réalisé par la mère. Le dispositif tel qu'il est actuellement configuré, produit des sentiments d'insécurité et de peur pour André, et un sentiment d'insatisfaction pour ses parents. Mais en même temps, cet agencement apporte à André un certain confort : une matérialité commune est formée, sur laquelle il peut s'appuyer pour faire certaines choses. Mettre en question cette matérialité commune, c'est risquer de tout perdre, ou, du moins, c'est perdre le confort qu'il a actuellement. D'où le refus et la peur d'André de changer de fauteuil.

Le processus d'ajustement est un processus ambivalent puisqu'il est à la fois ouverture et fermeture de la personne. A travers l'ajustement, ce que la personne peut être, faire, sentir... est à la fois mis en question et défini. Des possibilités sont ouvertes et d'autres fermées pour elle. L'ajustement produit une matérialité commune qui présente deux aspects. D'une part, elle est rendue rigide, cette rigidité est ce qui fait sa solidité sur laquelle la singularité peut s'appuyer pour agir, cette rigidité est aussi ce qui fixe la singularité dans un certain monde ; cette fixation peut être source de douleur ou de confort. D'autre part, elle est rendue souple, cette souplesse est ce qui permet de mettre en question certains liens qui font la singularité, et ce qui permet que cette fixité ne devienne pas fermeture; la souplesse est ce qui ouvre certaines possibilités nouvelles à la singularité. Ce processus à travers lequel la personne est fabriquée est ce que j'appelle le processus d'habilitation, en me référant à la différence opérée par le français entre « habileté » et «habilité ». L'habileté désigne la capacité, ce que l'on peut faire, l'habilité désigne la qualité qui rend apte. L'analyse de l'essai de fauteuil permet de montrer que l'acquisition d'un «faire », de capacités et d'incapacités, suppose l'émergence d'un être, c'est-à-dire une transformation de ce que la personne est, de son corps et de son monde. Le processus d'habilitation repose sur un long travail d'ajustement, sur une négociation entre la personne et les dispositifs, ici les dispositifs techniques. En outre, l'habilitation est aussi déshabilitation : acquérir certaines capacités, c'est en perdre d'autres.

\section{Conclusion}

La sociologie de la médecine a décrit l'expérience des personnes atteintes d'une maladie chronique ou ayant subi un accident comme une 
expérience touchant le soi ou l'identité, et comme une expérience mettant en cause le sens de l'existence de la personne. A travers cette description, elle a mis l'accent sur l'aspect cognitif et intime de cette expérience. Dans cet article, j'ai décrit cette expérience en mettant l'accent sur la transformation corporelle vécue par la personne. Cette analyse m'a permis d'accorder une place centrale au corps et au processus d'embodiment, puisque j'ai montré comment le corps et le monde de la personne étaient transformés dans un même mouvement, soit de rétraction, soit d'extension. La maladie et l'interaction avec le dispositif d'aide sont apparues comme des moments au cours desquels ce qu'est la personne est problématisé et fabriqué. L'une des questions sur lesquelles débouche directement cette analyse est la suivante : dans quelle mesure la description proposée est-elle généralisable à d'autres pathologies ? Comme je l'ai dit dans l'introduction, même si dans le centre d'essai, j'ai pu observer des pathologies diverses, ma recherche s'est essentiellement focalisée sur les personnes atteintes d'une maladie neuromusculaire dans lesquelles le corps est touché d'une manière très particulière. La notion de rétraction est issue de l'analyse de mes rencontres avec ces personnes. De manière plus large, cette analyse ouvre sur deux questions. La première concerne la notion de personne et la seconde concerne l'analyse du handicap. L'analyse entamée ici permet en premier lieu de poser la question de ce qu'est la personne (Douglas, 1999; Mauss, 1950a; Novaes, 1991). L'hypothèse, dans cet article, est que la personne est fabriquée à travers l'interaction avec différents dispositifs dont le dispositif technique étudié ici. J'ai montré ailleurs (Winance, 2001) l'importance du dispositif discursif et du dispositif institutionnel. Les qualités et les capacités de la personne - ses dispositions - sont définies dans ces interactions. L'enjeu de cette analyse est théorique et politique. L'enjeu théorique est d'arriver à proposer une voie intermédiaire entre les deux conceptions opposées de la personne: comme donnée (essentialisme, philosophie personnaliste) ou construite (Baud, 1993 et 2001). L'enjeu politique est d'arriver à reposer la question de «la reconnaissance de la personne» sous la forme : «Comment les acteurs, dans leurs pratiques, fabriquent/ performent-ils la personne ?».

Deuxièmement, cette analyse de la fabrication de la personne permet de repenser la question du handicap. Dans la littérature, le handicap est soit défini comme une différence absolue et essentielle (Stiker, 1997), soit comme une différence accidentelle et relative causée par l'environnement physico-humain (conception selon laquelle on est tous plus ou moins capables) (Moser et Law, 1999). Dans la première optique, la personne handicapée perd ce qui la rend même par rapport aux valides, dans la seconde, elle perd ce qui la rend différente par rapport à eux. L'analyse du 
processus d'habilitation pourrait permettre de penser comment la personne handicapée, comme la personne valide, est fabriquée comme même (normale) et différente à travers les interactions avec des dispositifs ou des humains.

\section{REFERENCES BIBLIOGRAPHIQUES}

Akrich M., 1987, Comment décrire les objets techniques ? Technique et culture, 9, 49-64.

Baud J.P., 1993, L’affaire de la main volée. Une histoire juridique du corps, Paris, Seuil.

Baud J.P., 2001, Le droit de vie et de mort. Archéologie de la bioéthique, Paris, Aubier.

Baszanger, I. (1986). Les maladies chroniques et leur ordre négocié, Revue Française de Sociologie, XXVII, 3-27.

Bury M., 1982, Chronic illness as biographical disruption, Sociology of Health and Illness, IV, 2, 167-182.

Callon M., 1986, Eléments pour une sociologie de la traduction. La domestication des Coquilles Saint-Jacques et des marins-pêcheurs de la Baie de Saint-Brieuc, in L'Année Sociologique, vol 36, PUF, Paris, 171-208.

Callon M., Rabeharisoa V., à paraître, Articulating bodies : The Case of Muscular Dystrophies, In : Akrich M., Berg M., eds, Bodies on Trial : Performances and Politics in Medecine and Biology, Body \& Society.

Charmaz K., 1983, Loss of self : a fundamental form of suffering in the chronically ill, Sociology of Health and Illness, V, 2, 168-195.

Charmaz K., 1999, The Body, Identity, and Self: Adapting to Impairment, In : Charmaz K., Paterniti D. A., eds, Health, Illness, and Healing. Society, Social Context, and Self. An Anthology, , Los Angeles, Roxbury Publishing Company, 95-112.

Clarke A., 2000, Technosciences et nouvelle biomédicalisation : racines occidentales et rhizomes mondiaux, Sciences Sociales et Santé, 18, 2, 11-42.

Despret V., 1999, Ces émotions qui nous fabriquent. Ethnopsychologie de l'authenticité, Le Plessis-Robinson, Institut Synthélabo pour le progrès de la connaissance, Les Empêcheurs de penser en ronds.

Dodier N, 1993, Les arènes des habiletés techniques, In : Conein, B., Dodier, N., Thévenot, L., eds, Raisons Pratiques. Les objets dans l'action, 4, Paris, Ed. de l'EHESS, 115-139.

Dodier N., 1995, Les Hommes et les Machines, Paris, Métaillé. 
Douglas M., 1999, La connaissance de soi, In: Dougles, M., Comment pensent les institutions, Paris, La Découverte/M.A.U.S.S., 152-162.

Gomart E., Hennion A., 1999, A Sociology of Attachment : Music Amateurs and Drug Users, In : Law J., Hassard J., eds, Actor Network Theory and After, Oxford, Blackwell and the Sociological Review, 220-247.

Hirschauer S, 1991, The Manufacture of Bodies in Surgery, Social Studies of Science, XXI, 2, 279-319.

Kelly M., 1992, Self, Identity and Radical Surgery, Sociology of Health \& Illness, XIV, 3, 390-415.

Latour B., 1993, La clef de Berlin et autres leçons d'un amateur des sciences, Paris, La Découverte.

Latour B., 2000a, Factures/fractures : de la notion de réseau à celle d'attachement, In : Micoud A., Peroni M., eds, Ce qui nous relie, La tour d'Aigues, Editions de l'Aube, 189-208.

Latour B., 2000b, When things strike back : a possible contribution of 'science studies' to the social sciences, British Journal of Sociology, Vol 51, 1, 107-123.

Leder D., 1990, The absent Body, Chicago, University of Chicago Press.

Mauss M., 1950a, Une catégorie de l'esprit humain : la notion de personne, celle de « moi », In : Mauss M., Sociologie et anthropologie, Paris, PUF, 331-362.

Mauss M., 1950b, Les techniques du corps, Sociologie et anthropologie, Paris, PUF, 365386.

Merleau-Ponty M., 1971, La phénoménologie de la perception, Paris, Gallimard.

Morin C., Salazar-Orvig A., 1996, Paroles de patients hémiplégiques : discours et position subjective, Sciences Sociales et Santé, XIV, 2, 47-77.

Moser I., Law J., 1999, Good Passages, Bad Passages, In : Law J., Hassard J., eds, Actor Network Theory and After, Oxford and Keele, Blackwell and the Sociological Review, 196219.

Murphy R. F., 1990, The Body Silent. An anthropologist embarks on the most challenging journey of his life : into the world of the disabled, New York - London, W.W. Norton.

Novaes S., 1991, Biomédecine et devenir de la personne, Paris, Le Seuil.

Rabeharisoa V., Callon M., 2000, De la médiation, ou les enjeux d'un nouveau métier dans le secteur de la prise en charge des personnes handicapées, Contraste. Enfance et Handicap, 13, 217-235.

Scarry E., 1985, The Body in Pain. The Making and unmaking of the World, New York et Oxford, Oxford University Press. 
Seymour W., 1998, Remaking the Body. Rehabilitation and Change, London and New York, Routledge.

Stiker H.J., 1997, Corps infirmes et sociétés, Paris, Dunod.

Strauss A., 1992, La trame de la négociation. Sociologie qualitative et interactionnisme. Textes réunis et présentés par Isabelle Baszanger, Paris, L'Harmattan.

Suchman L., 2002, Replicants and Irreductions : Affective encounters at the interface, Paper presented to the European Association of the Study of Science and Technology (EASST), York, UK, 2 August 2002, published by the department of Sociology, Lancaster University at : http://www.comp.lancs.ac.uk/sociology/soc0391s.html

Thévenot L., 1994, Le régime de la familiarité. Les choses en personne, Genèse, 17, 72-101.

Ville, I., Ravaud, J.-F., 1994, Représentations de soi et traitement social du handicap. L'intérêt d'une approche socio-constructiviste, Sciences Sociales et Santé, XII, 1, 7-30.

Waissman R., 1991, L'enfant, la famille et la maladie chronique : construction d'une autonomie, In : S. Novaes, dir., Biomédecine et devenir de la personne, Paris, Seuil, 290-313.

Waissman R., 1995, Interactions familiales et impact de la technologie dans la gestion d'une maladie chronique chez l'enfant, Sciences Sociales et Santé, XIII, 1, 81-99.

Williams S.J. et G. Bendelow, 1998, The lived body. Sociological Themes, Embodies Issues, London and New York, Routledge.

Winance M., 2000, De l'ajustement entre les prothèses et les personnes : interactions et transformations mutuelles, Handicap, revue de sciences humaines et sociales, 85, 11-26.

Winance M., 2001, Thèse et Prothèse. Le processus d'habilitation comme fabrication de la personne. L'Association Française contre les Myopathies face au handicap, Centre de Sociologie de l'Innovation, Paris, Ecoles Nationale des Mines de Paris.

Yoshida K., 1993, Reshaping of self: a pendular reconstruction of self and identity among adults with traumatic spinal cord injury, Sociology of Health \& Illness, 15, 2, 217-245. 